Cahiers $d u$ MONDE RUSSE

\section{Cahiers du monde russe}

Russie - Empire russe - Union soviétique et États indépendants

50/2-3 | 2009

L'Europe orientale, 1650-1730. Crises, conflits et renouveau

\title{
Ghostly ParadoxesIlya VINITSKY
}

, Toronto : University of Toronto Press, 2009, 252 p.

\section{Мария Майофис}

\section{(2) OpenEdition}

\section{Journals}

Édition électronique

URL : https://journals.openedition.org/monderusse/9754

DOI : 10.4000/monderusse.9754

ISSN : $1777-5388$

Éditeur

Éditions de l'EHESS

Édition imprimée

Date de publication : 15 septembre 2009

ISBN : 978-2-7132-2260-3

ISSN : $1252-6576$

Référence électronique

Мария Майофис, " Ghostly Paradoxesllya VINITSKY », Cahiers du monde russe [En ligne], 50/2-3 |

2009, mis en ligne le 14 janvier 2013, consulté le 03 septembre 2022. URL : http://

journals.openedition.org/monderusse/9754; DOI : https://doi.org/10.4000/monderusse.9754

Ce document a été généré automatiquement le 3 septembre 2022

Tous droits réservés 


\title{
Ghostly ParadoxesIlya VINITSKY
}

, Toronto : University of Toronto Press, 2009, 252 p.

\author{
Мария Майофис
}

\section{Ilya VINITSKY, Ghostly Paradoxes, Modern Spiritualism and Russian Culture in the Age of Realism, Toronto : University of Toronto Press, 2009, $252 \mathrm{p}$.}

1 «Ghostly Paradoxes» - НОВАЯ КНИГА ИСТОРИКА ЛИТЕРАТУРЫ И КУЛЬТУРЫ ИЛЬИ ВИНИЦКОГО, ПРОФЕССОРА УНИВЕРСИТЕТА ПЕНСИЛЬВАНИИ (ФИЛАДЕЛЬФИЯ). ЭТО ОДНА ИЗ САМЫХ ОРИГИНАЛЬНЫХ И НОВАТОРСКИХ КНИГ ПО ИСТОРИИ РУССКОЙ КУЛЬТУРЫ ХІХ ВЕКА ИЗ ЧИСЛА ПОЯВИВШИХСЯ В ПОСЛЕДНИЕ НЕСКОЛЬКО ЛЕТ.

ИССЛЕДОВАТЕЛЬСКАЯ СТРАТЕГИЯ, ПРИМЕНЕННАЯ В ЭТОЙ КНИГЕ, БЛИЗКА К ТОЙ, ЧТО ОПИСАЛ МНОГО ЛЕТ НАЗАД В СВОЕМ МАНИФЕСТАРНОМ ПРЕДИСЛОВИИ КАРЛО ГИНЗБУРГ: «...РЕЧЬ ШЛА О ТОМ, ЧТОБЫ ВВЕСТИ В СФЕРУ ИСТОРИЧЕСКОГО ПОЗНАНИЯ НЕ ТЕ ФЕНОМЕНЫ, КОТОРЫЕ КАЖУТСЯ ВНЕВРЕМЕННЫМИ, А ТЕ, КОТОРЫЕ КАЖУТСЯ НЕСУЩЕСТВЕННЫМИ <..> НО ЧТОБЫ ПРОДЕМОНСТРИРОВАТЬ ЗНАЧИМОСТЬ НЕСУЩЕСТВЕННЫХ, НА ПЕРВЫЙ ВЗГЛЯД, ЯВЛЕНИЙ, НЕОБХОДИМО БЫЛО ПРИБЕГНУТЬ К НОВЫМ ИССЛЕДОВАТЕЛЬСКИМ ИНСТРУМЕНТАМ И К НОВЫМ МАСШТАБАМ НАБЛЮДЕНИЯ, ОТЛИЧАЮЩИМСЯ ОТ ПРИВЫЧНЫХ». ИЛЬЯ ВИНИЦКИЙ ПРЕДЛАГАЕТ И ОБОСНОВЫВАЕТ В СВОЕЙ КНИГЕ ИМЕННО ТАКУЮ СМЕНУ МАСШТАБОВ И ИНСТРУМЕНТАРИЯ. «НЕСУЩЕСТВЕННЫМ» ОБЪЕКТОМ, КОТОРЫЙ ПОЗВОЛИЛ ВИНИЦКОМУ ЗАНОВО РАССМОТРЕТЬ ИСТОРИЮ РУССКОЙ КУЛЬТУРЫ ВТОРОЙ ПОЛОВИНЫ ХІХ ВЕКА, СТАЛ СПИРИТИЗМ КАК РАЗВЕТВЛЕННЫЙ КОМПЛЕКС ПРАКТИК И АРТЕФАКТОВ: СОБСТВЕННО ВЫЗЫВАНИЕ ДУХОВ И СКЛАДЫВАЮЩИЕСЯ ВОКРУГ СПИРИТИЧЕСКИХ СЕАНСОВ СООБЩЕСТВА; ТЕКСТЫ, КОТОРЫЕ, КАК СЧИТАЛОСЬ, БЫЛИ «ПРОДИКТОВАНЫ» ДУХАМИ В ПРОЦЕССЕ СПИРИТИЧЕСКИХ СЕАНСОВ; ФИЛОСОФСКИЕ И ЕСТЕСТВЕННОНАУЧНЫЕ «ОБОСНОВАНИЯ» СПИРИТИЗМА; ДИСКУССИИ В ПЕРИОДИЧЕСКОЙ ПЕЧАТИ ПО ПОВОДУ СПИРИТИЧЕСКИХ СЕАНСОВ, ТЕКСТОВ И ДЕЯТЕЛЬНОСТИ ЗНАМЕНИТЫХ МЕДИУМОВ. 
КНИГА РАЗДЕЛЕНА НА ДВЕ ЧАСТИ. ПЕРВАЯ ПОСВЯЩЕНА СПИРИТИЧЕСКОМУ СЕАНСУ КАК КУЛЬТУРНОЙ МЕТАФОРЕ: В НЕЙ ПОСЛЕДОВАТЕЛЬНО РАССМОТРЕНЫ ИСТОРИЯ СОВМЕСТНОГО УЧАСТИЯ Ф.М. ДОСТОЕВСКОГО, Н.С. ЛЕСКОВА И П.Д. БОБОРЫКИНА В СПИРИТИЧЕСКОМ СЕАНСЕ, СОСТОЯВШЕМСЯ В ФЕВРАЛЕ 1876 ГОДА, И ОТЗЫВЫ ПИСАТЕЛЕЙ ОБ ЭТОМ ОПЫТЕ (ГЛ. 1), ИСТОРИЧЕСКАЯ ДРАМАТУРГИЯ И СОБСТВЕННО ИСТОРИОГРАФИЯ 1860-Х ГОДОВ КАК БЛИЗКИЕ (ПО МЕТОДУ ОБРАЩЕНИЯ К ПРОШЛОМУ) АНАЛОГИ СПИРИТИЧЕСКОГО СЕАНСА (ГЛ. 2) И, НАКОНЕЦ, ПРАКТИКИ ЗАПИСИ И РАСПРОСТРАНЕНИЯ ТЕКСТОВ, ЯКОБЫ НАДИКТОВАННЫХ ИЗВЕСТНЫМИ РУССКИМИ ПОЭТАМИ (ПУШКИНЫМ, ЛЕРМОНТОВЫМ, БАРКОВЫМ И ПРОч.) (ГЛ. 3).

ВТОРАЯ ЧАСТЬ КНИГИ ПОСВЯЩЕНА ВЗАИМООТНОШЕНИЯМ СПИРИТИЗМА И РУССКОЙ ХУДОЖЕСТВЕННОЙ ЛИТЕРАТУРЫ 1860-1880-Х ГОДОВ. ЕЕ ГЕРОЯМИ СТАЛИ ПИСАТЕЛЬ И УЧЕНЫЙ-ЕСТЕСТВОИСПЫТАТЕЛЬ НИКОЛАЙ ВАГНЕР (ГЛ. 4), Н.Е. САЛТЫКОВ-ЩЕДРИН (ГЛ. 5), Ф.М. ДОСТОЕВСКИЙ (гЛ. 6), Л.Н. ТОЛСТОЙ (гЛ. 7) И Н.С. ЛЕСКОВ (ГЛ. 8).

КАК СПРАВЕДЛИВО ПИШЕТ САМ ВИНИЦКИЙ, ДО НАЧАЛА 1990-Х ГОДОВ ИСТОРИКИ КУЛЬТУРЫ И ИСТОРИКИ ИДЕЙ СЧИТАЛИ РАЗНОГО РОДА «БЫТОВУЮ ДЕМОНОЛОГИЮ» ВТОРОЙ ПОЛОВИНЫ ХIX И ПЕРВОЙ ПОЛОВИНЫ ХX ВЕКА СОВЕРШЕННО НЕ ЗАСЛУЖИВАВШЕЙ ВНИМАНИЯ. ОДНАКО В ПОСЛЕДНИЕ ДВАДЦАТЬ ЛЕТ ПОЛОЖЕНИЕ ИЗМЕНИЛОСЬ: В КНИГАХ ЖАКА ДЕРРИДА «Spectres de Marx : l’État de la dette, le travail du deuil et la nouvelle Internationale» (1993), ТЕРИ кэсл «The Female Thermometer: Eighteenth-Century Culture and the Invention of the Uncanny» (1995), ЭВЕРИ Ф. ГОРДОН «Ghostly Matters: Haunting and the Sociological Imagination» (1996), СТИВЕНА ГРИНБЛАТТА «Нamlet in Purgatory» (2001) И ЭЛЕН СОРД «Ghostwriting Modernism» (2002) АВТОРЫ, ПО СЛОВАМ ВИНИЦКОГО, ТАК ИЛИ ИНАЧЕ ПОПЫТАЛИСЬ «“РАЗГОВОРИТЬ” ПРИВИДЕНИЕ, ЗАСТАВИТЬ ЕГО “РАССКАЗАТЬ” О ТЕХ РЕЛИГИОЗНЫХ, КУЛЬТУРНЫХ, ПСИХОЛОГИЧЕСКИХ, ИДЕОЛОГИЧЕСКИХ ИЛИ ЭСТЕТИЧЕСКИХ КОНФЛИКТАХ, КОТОРЫЕ ОНО “МАТЕРИАЛИЗУЕТ”» (С. ХІІІ).

КНИГА ВИНИЦКОГО ОРГАНИЧНО ВСТРАИВАЕТСЯ В КОНТЕКСТ ПЕРЕЧИСЛЕННЫХ ВЫШЕ РАБОТ - ОНА ВПЕРВЫЕ ПРЕДЛАГАЕТ МАСШТАБНУЮ ИСТОРИКО-КУЛЬТУРНУЮ РАЗРАБОТКУ «СПИРИТИЧЕСКОЙ» ТЕМЫ НА РОССИЙСКОМ МАТЕРИАЛЕ. ЭТО ТЕМ БОЛЕЕ ВАЖНО, ЧТО В ПОСЛЕДНИЕ ГОДЫ ПРАВЛЕНИЯ НИКОЛАЯ І СПИРИТИЗМ БЫЛ, КАК ПОКАЗЫВАЕТ ВИНИЦКИЙ, ЕДИНСТВЕННЫМ ИНОСТРАННЫМ ДУХОВНЫМ УЧЕНИЕМ, ПОЛУЧИВШИМ РАСПРОСТРАНЕНИЕ В РОССИИ, ХОТЯ БЫ И ПОЛУЛЕГАЛЬНОЕ (С. 5).

ОДНАКО ОРИГИНАЛЬНОСТЬ КНИГИ ОБУСЛОВЛЕНА НЕ ТОЛЬКО НОВИЗНОЙ МАТЕРИАЛА. ПО СРАВНЕНИЮ СО ВСЕМИ ПЕРЕЧИСЛЕННЫМИ ВЫШЕ РАБОТАМИ ВИНИЦКИЙ ПРИНЦИПИАЛЬНО ШИРОКО КОНСТРУИРУЕТ СВОЙ ИССЛЕДОВАТЕЛЬСКИЙ ОБЪЕКТ. В РАМКАХ ОДНОЙ КНИГИ ОБСУЖДАЕТСЯ И ОТРАЖЕНИЕ ПРОБЛЕМАТИКИ СПИРИТИЗМА В ПРОИЗВЕДЕНИЯХ ВЫДАЮЩИХСЯ РУССКИХ ПИСАТЕЛЕЙ, И ПОЛУГРАФОМАНСКИЕ СТИХИ, НАПИСАННЫЕ МЕДИУМАМИ ПОД ВОЗДЕЙСТВИЕМ «ИНСПИРАЦИЙ», ЯКОБЫ ИСХОДИВШИХ ОТ ДУХОВ УМЕРШИХ ПОЭТОВ. ЕЩЕ БОЛЕЕ ВАЖНОЕ - И, ПОЖАЛУЙ, ГЛАВНОЕ - ДОСТИЖЕНИЕ ЭТОЙ КНИГИ СОСТОИТ В ТОМ, ЧТО СПИРИТИЧЕСКИЕ ФЕНОМЕНЫ - СООБЩЕСТВА, ДИСКУССИИ, СОБСТВЕННО СПИРИТИЧЕСКИЕ СЕАНСЫ РАССМАТРИВАЮТСЯ КАК НЕОБХОДИМАЯ СОСТАВЛЯЮЩАЯ 1850-1870-Х ГОДОВ, - ЭПОХИ, КОТОРУЮ ДО СИХ ПОР ПРИНЯТО ОПИСЫВАТЬ КАК ПРЕДЕЛЬНО МАТЕРИАЛИСТИЧЕСКУЮ И ПОЗИТИВИСТСКУЮ ПО СВОЕМУ ОБЩЕМУ ИНТЕЛЛЕКТУАЛЬНОМУ НАСТРОЕНИЮ. 
ВПЛОТЬ ДО НАСТОЯЩЕГО ВРЕМЕНИ В ИСТОРИИ ЛИТЕРАТУРЫ СОХРАНЯЕТСЯ ПРЕДСТАВЛЕНИЕ О ТОМ, ЧТО РОМАНТИЗМ, РЕАЛИЗМ И МОДЕРНИЗМ ПРЕДСТАВЛЯЮТ СОБОЙ ПОСЛЕДОВАТЕЛЬНО СМЕНЯВШИЕ ДРУГ ДРУГА ЭСТЕТИЧЕСКИЕ СИСТЕМЫ, ВЗАИМОДЕЙСТВУЮЩИЕ ПО ПРИНЦИПУ «ТЕЗИС-АНТИТЕЗИС-СИНТЕЗ», ПРИЧЕМ В ЭТОЙ ЭВОЛЮЦИОННОЙ ЦЕПИ КАЖДАЯ СЛЕДУЮЩАЯ СТАДИЯ В СУЩЕСТВЕННЫХ ЧЕРТАХ ОТТАЛКИВАЕТСЯ ОТ ПРЕДЫДУЩЕЙ. ПОПЫТКИ ПЕРЕСМОТРЕТЬ ЭТУ СХЕМУ БЫЛИ ЛИБО ПЕРСОНАЛИСТИЧЕСКИМИ, ЛИБО ЭПАТАЖНО-СКЕПТИЦИСТСКИМИ. ПЕРСОНАЛИСТИЧЕСКИЕ ПОПЫТКИ СВОДИЛИСЬ К ЭСТЕТИЧЕСКОЙ КАНОНИЗАЦИИ АВТОРОВ, КРИТИЧЕСКИ «ПОДРЫВАВШИХ» ГОСПОДСТВОВАВШИЕ В «ЭПОХУ РЕАЛИЗМА» ПОЗИТИВИСТСКИЕ НАСТРОЕНИЯ - В СЛУЧАЕ РУССКОЙ ЛИТЕРАТУРЫ РЕЧЬ ИДЕТ ОБ АПОЛЛОНЕ ГРИГОРЬЕВЕ, АФАНАСИИ ФЕТЕ, АЛЕКСЕЕ К. ТОЛСТОМ, НИКОЛАЕ ЛЕСКОВЕ. В РОССИИ ЭТОТ ПУТЬ ИЗБРАЛИ МНОГИЕ КРИТИКИ И ИСТОРИКИ ЛИТЕРАТУРЫ 1970-1990-Х ГОДОВ ${ }^{10}$. С ЭПАТАЖНО-СКЕПТИЦИСТСКИХ ПОЗИЦИЙ ЭТУ СХЕМУ ПОПЫТАЛСЯ ПЕРЕСМОТРЕТЬ ФИЛОСОФ ВАДИМ РУДНЕВ - ОН ПРЕДЛОЖИЛ СЧИТАТЬ РЕАЛИЗМ» НЕ СЛИШКОМ УДАЧНЫМ НАЗВАНИЕМ ДЛЯ ИСКУССТВА ПОЗДНЕГО РОМАНТИЗМА (ПО АНАЛОГИИ С ТЕМ, КАК ВТОРАЯ ПОЛОВИНА ХІХ ВЕКА В ИСТОРИИ СИМФОНИЧЕСКОЙ МУЗЫКИ СЧИТАЕТСЯ ПЕРИОДОМ ПОЗДНЕГО РОМАНТИЗМА $)^{11}$.

ВИНИЦКИЙ РАВНО ДАЛЕК ОТ ЭТИХ КРАЙНОСТЕЙ - «ПАРТИЗАНСКОЙ» И «ОПРОВЕРГАЮЩЕЙ». ОН ПОКАЗЫВАЕТ, ЧТО РЕАЛИЗМ ИМЕННО В СВОИХ СУЩНОСТНЫХ ЧЕРТАХ, В УСТРЕМЛЕНИЯХ К РЕПРЕЗЕНТАЦИИ «ПОТУСТОРОННЕЙ», ФИКЦИОНАЛЬНОЙ РЕАЛЬНОСТИ КАК РЕАЛЬНОСТИ ЭТОГО МИРА БЫЛ ЯВЛЕНИЕМ ТОГО ЖЕ ПОРЯДКА, ЧТО И СПИРИТИЗМ. ТАКИМ ОБРАЗОМ, РЕАЛИЗМ И СПИРИТИЗМ В РАВНОЙ СТЕПЕНИ НАСЛЕДОВАЛИ РОМАНТИЗМУ, И РЕАЛИЗМ - В ГОРАЗДО БОЛЬШЕЙ СТЕПЕНИ, ЧЕМ КАЗАЛОСЬ РАНЕЕ.

10 НО У ЭТОГО ПРОЦЕССА БЫЛА И ДРУГАЯ СТОРОНА: СПИРИТИЗМ, КАК ПОКАЗЫВАЕТ ВИНИЦКИЙ, В ЗНАЧИТЕЛЬНОЙ СТЕПЕНИ ОРИЕНТИРОВАЛСЯ НА НОРМЫ И КОНЦЕПЦИИ ПОЗИТИВНОЙ НАУКИ. В ОСНОВЕ СПИРИТИЗМА ЛЕЖАЛО ЖЕЛАНИЕ РАЗРЕШИТЬ НАИБОЛЕЕ ЗАХВАТЫВАЮЩИЕ ИСТОРИЧЕСКИЕ И МЕТАФИЗИЧЕСКИЕ ЗАГАДКИ С ПОМОЩЬЮ ПРОЦЕДУР ЭКСПЕРИМЕНТАЛЬНОГО ЕСТЕСТВОЗНАНИЯ. ЭТО ОБУСЛОВИЛО ДРАМАТИЗМ ПОЛОЖЕНИЯ СПИРИТОВ, ОСОБЕННО ОСТРО ПРОЯВИВШИЙСЯ В РОССИЙСКОЙ СИТУАЦИИ ПЕРИОДА ВЕЛИКИХ РЕФОРМ: ИХ КРИТИКОВАЛИ И СТОРОННИКИ «НОРМАЛЬНОГО» ЕСТЕСТВОЗНАНИЯ (ОНИ ТРАКТОВАЛИ СПИРИТИЧЕСКИЙ ОПЫТ КАК КОЛЛЕКТИВНУЮ ГАЛЛЮЦИНАЦИЮ), И СТОРОННИКИ ОФИЦИАЛЬНОЙ ЦЕРКВИ (ОНИ СЧИТАЛИ СПИРИТИЗМ СЛЕДСТВИЕМ МАЛОВЕРИЯ).

11 НОВАЯ КНИГА ОТЧАСТИ ПРОДОЛЖАЕТ ПРЕЖНИЕ РАБОТЫ ВИНИЦКОГО - «НЕЧТО О ПРИВИДЕНИЯХ» (1998) И «УТЕХИ МЕЛАНХОЛИИ» (1997), В КОТОРЫХ ШЛА РЕЧЬ О «НИЗШЕЙ ДЕМОНОЛОГИИ» И ПЕРСОНИФИКАЦИЯХ ПСИХИЧЕСКИХ СИЛ В ТВОРЧЕСТВЕ В.А. ЖУКОВСКОГО И Н.В. ГОГОЛЯ ${ }^{12}$. ОДНАКО ЭТИ КНИГИ БЫЛИ МЕНЕЕ РАДИКАЛЬНЫМИ ПО СВОИМ ВЫВОДАМ, ЧЕМ «Ghostly Paradoxes». В САМОМ ДЕЛЕ, ИНТЕРЕС ПИСАТЕЛЕЙРОМАНТИКОВ К ОПИСАНИЮ НЕМАТЕРИАЛЬНЫХ, ПОТУСТОРОННИХ СУЩНОСТЕЙ ЛОГИЧНО СЛЕДОВАЛ ИЗ ОБЩИХ ЧЕРТ РОМАНТИЧЕСКОЙ ЭСТЕТИЧЕСКОЙ ПРОГРАММЫ ВО ВСЕХ ЕЕ МНОГОЧИСЛЕННЫХ ВАРИАНТАХ: ОБРАЩЕНИЯ К ФОЛЬКЛОРУ И СРЕДНЕВЕКОВЬЮ, ПОЛЕМИКИ С РАЦИОНАЛИЗМОМ ПРОСВЕЩЕНИЯ, ВНИМАНИЯ КО ВСЕМУ ТАИНСТВЕННОМУ И НЕПОСТИЖИМОМУ. НОВАЯ КНИГА ВИНИЦКОГО 
ПОКАЗЫВАЕТ, ЧТО РЕАЛИСТЫ ИСПЫТЫВАЛИ НЕ МЕНЬШУЮ, ХОТЯ И ИНУЮ ПО СВОЕМУ ВЫРАЖЕНИЮ, «ЗАВОРОЖЕННОСТЬ ПОТУСТОРОННИМ», ЧЕМ РОМАНТИКИ.

ИСТОРИЯ ИНТЕРЕСА «РЕАЛИСТОВ» К ПОТУСТОРОННЕМУ И ЯВЛЯЕТСЯ ОДНИМ ИЗ ГЛАВНЫХ СЮЖЕТОВ КНИГИ. ПРЕДСТАВЛЕНИЕ О ДУХОВНЫХ СИЛАХ В 1850-Е ГОДЫ ПО СРАВНЕНИЮ С 1830-МИ СТАНОВИТСЯ БОЛЕЕ ДЕМОКРАТИЧЕСКИМ И БОЛЕЕ «ЭКСПЕРИМЕНТАЛЬНЫМ». СОЗЕРЦАНИЕ ДУХОВ В КУЛЬТУРЕ РОМАНТИЗМА - УДЕЛ ИЗБРАННЫХ, НАДЕЛЕННЫХ ОСОБЫМИ СПОСОБНОСТЯМИ НАТУР. «В 1830-1840-Е ГОДЫ ТЕМА ПРИВИДЕНИЙ АССОЦИИРОВАЛОСЬ С ПОПУЛЯРНЕЙШЕЙ ТЕМОЙ ЯСНОВИДЕНИЯ, ТО ЕСТЬ СПОСОБНОСТИ НЕКОТОРЫХ ЛЮДЕЙ... ПРОНИКАТЬ В СОСТОЯНИИ СОМНАМБУЛИЧЕСКОГО ТРАНСА В ПРОШЛОЕ ИЛИ В БУДУЩЕЕ, ЧИТАТЬ МЫСЛИ НА РАССТОЯНИИ, ВИДЕТЬ ДУХОВ И БЕСЕДОВАТЬ С НИМИ» ${ }^{13}$. В 1850-1860-Е ГОДЫ ОБЩЕНИЕ С ПОТУСТОРОННИМ МИРОМ ОКАЗЫВАЕТСЯ ПРЕЖДЕ ВСЕГО РЕЗУЛЬТАТОМ ПРИМЕНЕНИЯ «НАУЧНО» ОБОСНОВАННЫХ МЕТОДОВ. СПИРИТИЧЕСКИЙ СЕАНС ВИНИЦКИЙ ОПИСЫВАЕТ КАК «МИРАКЛЬ», ПО СВОИМ ФИЛОСОФСКИМ ПРЕДПОСЫЛКАМ И СОЦИАЛЬНЫМ ХАРАКТЕРИСТИКАМ НАХОДЯЩИЙСЯ ПОСЕРЕДИНЕ МЕЖДУ НАУЧНЫМИ И РЕЛИГИОЗНЫМИ ПРАКТИКАМИ. АКТЕРЫ В НЕМ - «И МЕДИУМЫ, И ЗРИТЕЛИ, А ДЕЙСТВИЕ ПРОИСХОДИТ НА ГРАНИЦЕ ФИЗИЧЕСКОГО И ДУХОВНОГО МИРА» (С. 62).

СПИРИТИЗМ И РЕАЛИЗМ, ТАКИМ ОБРАЗОМ, ОКАЗЫВАЮТСЯ РАВНОПРАВНЫМИ ВЫРАЖЕНИЯМИ СОЦИАЛЬНО-ПСИХОЛОГИЧЕСКИХ ИЗМЕНЕНИЙ, ПРОИСХОДИВШИХ В РОССИИ 1850-1870-Х ГОДОВ. ВИНИЦКИЙ, ОДНАКО, НЕ ПИШЕТ О ТОМ, КАК В ЦЕЛОМ МОЖНО БЫЛО БЫ ОХАРАКТЕРИЗОВАТЬ ЭТИ ИЗМЕНЕНИЯ И БЫЛИ ЛИ У НИХ И ДРУГИЕ СТОЛЬ ЖЕ МАСШТАБНЫЕ КУЛЬТУРНЫЕ РЕПРЕЗЕНТАЦИИ.

СПИРИТИЗМ В КНИГЕ «Ghostly Paradoxes» - НЕ ТОЛЬКО ИССЛЕДОВАТЕЛЬСКИЙ ОБЪЕКТ, НО И МЕТОДОЛОГИЧЕСКАЯ «ЛИНЗА», СКВОЗЬ КОТОРУЮ ВИНИЦКИЙ РАССМАТРИВАЕТ ПРОТИВОРЕЧИЯ В ПРОИЗВЕДЕНИЯХ ПИСАТЕЛЕЙ-РЕАЛИСТОВ. О ТОМ, ЧТО МЕТОД «РЕАЛИЗМА» ОСНОВАН НА НЕЯВНОМ ПРОТИВОРЕЧИИ, ПРОНИЦАТЕЛЬНЫЕ ИССЛЕДОВАТЕЛИ ПИСАЛИ И РАНЬШЕ ${ }^{14}$, НО С ПОМОЩЬЮ ОПТИКИ, ИСПОЛЬЗОВАННОЙ ВИНИЦКИМ, АНАЛИЗ ЭТИХ ПРОТИВОРЕЧИЙ ОКАЗЫВАЕТСЯ ОСОБЕННО ЭФФЕКТИВНЫМ. ОТНОШЕНИЕ К СПИРИТИЗМУ ПРЕДСТАЕТ В КНИГЕ КАК - ПОЛЬЗУЯСЬ ПСИХОЛОГИЧЕСКИМ ТЕРМИНОМ - ПРОЕКТИВНЫЙ ТЕСТ, ПОЗВОЛЯЮЩИЙ СУДИТЬ О ГЛУБИННЫХ - ВПРОЧЕМ, ДАЛЕКО НЕ ВСЕГДА БЕССОЗНАТЕЛЬНЫХ - ОСНОВАХ ПОЭТИКИ НАИБОЛЕЕ ЗНАЧИТЕЛЬНЫХ РУССКИХ ПИСАТЕЛЕЙ ВТОРОЙ ПОЛОВИНЫ ХІХ ВЕКА. ТАК, НАПРИМЕР, ВИНИЦКИЙ ОБНАРУЖИВАЕТ, ЧТО КЛЮЧЕВОЕ ДЛЯ ЭСТЕТИЧЕСКИХ ТЕОРИЙ Л. ТОЛСТОГО 1880-1890-Х ГОДОВ ПОНЯТИЕ «ЗАРАЖЕНИЕ» ПОЯВЛЯЕТСЯ ПОД ВЛИЯНИЕМ ДИСКУССИЙ О ПСИХИЧЕСКОЙ ИНФЕКЦИИ И МАССОВОМ ГИПНОЗЕ.

ДВА ТИПА ИНСТРУМЕНТАРИЯ, ИСПОЛЬЗОВАННЫЕ ВИНИЦКИМ В ЕГО НОВОЙ КНИГЕ: ИСТОРИКО-ПОНЯТИЙНЫЙ - ПРИМЕНИТЕЛЬНО К ТВОРЧЕСТВУ КЛАССИКОВ РУССКОЙ ЛИТЕРАТУРЫ ВТОРОЙ ПОЛОВИНЫ ХІХ ВЕКА, И ЛИТЕРАТУРНО-СОЦИОЛОГИЧЕСКИЙ ПРИМЕНИТЕЛЬНО К МАССОВОЙ ПРОДУКЦИИ ТОЙ ЖЕ ЭПОХИ, - ПРИВОДЯТ АВТОРА К ОЧЕНЬ СМЕЛЫМ И В ТО ЖЕ ВРЕМЯ УБЕДИТЕЛЬНЫМ ВЫВОДАМ. ОДНАКО ПАРАЛЛЕЛЬНОЕ И ДАЖЕ ПОЧТИ СИНХРОННОЕ ИСПОЛЬЗОВАНИЕ ЭТИХ ИНСТРУМЕНТОВ ЗАСТАВЛЯЕТ ЗАДУМАТЬСЯ О ГРАНИЦАХ ПРИМЕНИМОСТИ ПО КРАЙНЕЙ МЕРЕ ВТОРОГО, СОЦИОЛОГИЧЕСКОГО ПОДХОДА.

НЕВОЗМОЖНО УСТАНОВИТЬ, НАСКОЛЬКО ТЕКСТУАЛЬНЫЕ ПРАКТИКИ (ТАКИЕ, КАК «АВТОМАТИЧЕСКОЕ ПИСЬМО» МЕДИУМОВ) И ОСОБЕННО ПРОИЗВЕДЕНИЯ «ВЫСОКОЙ» 
ЛИТЕРАТУРЫ, В КОТОРЫХ ОПИСЫВАЮТСЯ СПИРИТИЧЕСКИЕ СЕАНСЫ ИЛИ ИЗОБРАЖАЮТСЯ ПОКЛОННИКИ СПИРИТИЗМА, ПОЗВОЛЯЮТ СУДИТЬ О ПЕРЕЖИВАНИЯХ «РЯДОВЫХ» УЧАСТНИКОВ СПИРИТИЧЕСКОГО ДВИЖЕНИЯ. ОДНАКО РЕКОНСТРУКЦИЯ ЭТИХ ПЕРЕЖИВАНИЙ НЕОБХОДИМА ДЛЯ БОЛЕЕ ПОЛНОГО ПОНИМАНИЯ ТОЙ НОВОЙ КАРТИНЫ ИСТОРИКО-КУЛЬТУРНОГО ПРОЦЕССА, КОТОРУЮ ФОРМИРУЕТ ВИНИЦКИЙ. СУДЯ ПО ЕГО КНИГЕ, СРЕДНИЙ УРОВЕНЬ ЛИТЕРАТУРНОГО МАСТЕРСТВА, ЛИТЕРАТУРНЫХ СПОСОБНОСТЕЙ И ОСОБЕННО ПСИХОЛОГИЧЕСКОЙ РЕФЛЕКСИИ «ОБЫЧНЫХ», НЕ СВЯЗАННЫХ С ЛИТЕРАТУРОЙ ПОСЛЕДОВАТЕЛЕЙ СПИРИТИЗМА БЫЛ ВЕСЬМА НИЗКИМ, И ВМЕСТО ВЕРБАЛИЗАЦИИ СОБСТВЕННОГО МИРООТНОШЕНИЯ В ПРОЦЕССЕ «АВТОМАТИЧЕСКОГО ПИСЬМА» ОНИ В ЛУЧШЕМ СЛУЧАЕ МОГЛИ СТАРАТЕЛЬНО ВОСПРОИЗВОДИТЬ КУЛЬТУРНЫЕ ШТАМПЫ. ПОПЫТКА ПРОНИКНУТЬ В МИР РЯДОВЫХ» СПИРИТОВ НАТАЛКИВАЕТСЯ НА ТАКИЕ ЖЕ ТРУДНОСТИ, С КОТОРЫМИ ВСТРЕЧАЮТСЯ ИССЛЕДОВАТЕЛИ ЕВРОПЕЙСКОГО СРЕДНЕВЕКОВЬЯ И ДРУГИХ КУЛЬТУР, ОСНОВАННЫХ НА ИСПОЛЬЗОВАНИИ КЛИШЕ, СМЫСЛОВЫЕ ОТТЕНКИ КОТОРЫХ БЫЛИ ДОСТУПНЫ ТОЛЬКО САМИМ УЧАСТНИКАМ СОБЫТИЙ. ВОПРОС О СООТНОШЕНИИ ТЕКСТУАЛЬНЫХ ПРАКТИК И ЭМОЦИОНАЛЬНОЙ И МЕНТАЛЬНОЙ КАРТИНЫ МИРА РУССКИХ СПИРИТОВ ХІХ ВЕКА, КОНЕЧНО, ВЫХОДИТ ЗА РАМКИ ПОСТАВЛЕННОЙ АВТОРОМ ЗАДАЧИ И ТРЕБУЕТ ИЗУЧЕНИЯ МНОЖЕСТВА ДОПОЛНИТЕЛЬНЫХ (ПРЕЖДЕ ВСЕГО АРХИВНЫХ) ИСТОЧНИКОВ - ЭТО УЖЕ ТЕМА ДЛЯ СЛЕДУЮЩЕЙ КНИГИ.

НОВАЯ РАБОТА ВИНИЦКОГО ПОЗВОЛЯЕТ ПОСТАВИТЬ ЕЩЕ ОДИН ВОПРОС ОБЩЕГО ХАРАКТЕРА: ЯВЛЯЕТСЯ ЛИ ВНЕКОНФЕССИОНАЛЬНЫЙ ИНТЕРЕС К ПОТУСТОРОННИМ СИЛАМ ПРОСТЫМ СЛЕДСТВИЕМ ОЧЕНЬ СИЛЬНОГО - ГОРАЗДО БОЛЕЕ ЗНАЧИТЕЛЬНОГО, ЧЕМ КАЗАЛОСЬ РАНЕЕ - ВЛИЯНИЯ РОМАНТИЗМА НА РЕАЛИЗМ И ПОСЛЕДУЮЩИЕ ЭСТЕТИЧЕСКИЕ СИСТЕМЫ ИЛИ ЭТО ОДИН ИЗ ПОСТОЯННО ДЕЙСТВУЮЩИХ ВНУТРЕННИХ МЕХАНИЗМОВ ЕВРОПЕЙСКОЙ КУЛЬТУРЫ ЭПОХИ МОДЕРНОСТИ, КОГДА НАЧАЛОСЬ ФОРМИРОВАНИЕ СВОЕГО РОДА «ТЕНЕВЫХ» ФЕНОМЕНОВ РАЦИОНАЛЬНОСТИ, ХАРАКТЕРНОЙ ДЛЯ ЭПОХИ ПРОСВЕЩЕНИЯ? ${ }^{15}$ НАСКОЛЬКО МОЖНО СУДИТЬ, И ВИНИЦКИЙ, И АВТОРЫ ДРУГИХ, НАПИСАННЫХ В ПОСЛЕДНИЕ ДВА ДЕСЯТИЛЕТИЯ РАБОТ, ПОСВЯЩЕННЫХ ТЕМЕ «ПРИВИДЕНИЙ» СКОРЕЕ СКЛОНЯЮТСЯ КО ВТОРОМУ ОТВЕТУ.

В ЗАКЛЮЧЕНИЕ СЛЕДУЕТ ОТМЕТИТЬ, ЧТО НОВАЯ КНИГА ВИНИЦКОГО, НАПИСАННАЯ ПОАНГЛИЙСКИ, ОТЛИЧАЕТСЯ ТОЙ ИЗЯЩНОСТЬЮ СТИЛЯ И ОРИГИНАЛЬНОСТЬЮ КОМПОЗИЦИИ СТИЛЕМ, ЧТО И ЕГО РУССКИЕ РАБОТЫ, И ДЕМОНСТРИРУЕТ БОЛЬШОЕ МАСТЕРСТВО В ЧЕТКОМ И УБЕДИТЕЛЬНОМ АНАЛИЗЕ СТОЛЬ ТРУДНО УЛОВИМОГО ОПЫТА, КАК ОБЩЕНИЕ С ЗАГРОБНЫМИ ДУХАМИ.

\section{NOTES}

9. К. ГИНЗБУРГ, МИФЫ - ЭМБЛЕМЫ - ПРИМЕТЫ: МОРФОЛОГИЯ И ИСТОРИЯ, М.: НОВОЕ ИЗДАТЕЛЬСТВО, 2004, С. 13-14 (ПЕР. С ИТ. С.Л. КОЗЛОВА, С. Ginzburg, Miti, emblemi, spie: morfologia e storia, Torino : Einaudi, 1986). 
10. СМ., НАПРИМЕР: Л. АННИНСКИЙ, ЛЕСКОВСКОЕ ОЖЕРЕЛЬЕ, М. : КНИГА,1982 (2-Е ИЗД., 1986) ; А.С. НЕМЗЕР, « АЛЕКСЕЙ КОНСТАНТИНОВИЧ ТОЛСТОЙ », ПАМЯТНЫЕ ДАТЫ, М.: ВРЕМЯ, 2002, И ДРУГИЕ РАБОТЫ.

11. В. РУДНЕВ, МОРФОЛОГИЯ РЕАЛЬНОСТИ, М. : ГНОЗИС, 1996.

12. Я СОЗНАТЕЛЬНО ОСТАВЛЯЮ В СТОРОНЕ ДРУГИЕ СЮЖЕТЫ ЭТИХ КНИГ, СВЯЗАННЫЕ С ТВОРЧЕСТВОМ В.Л. ПУШКИНА, ВЛИЯНИЕМ И.Г. ЮНГА-ШТИЛЛИНГА НА РУССКУЮ ЛИТЕРАТУРУ И ДР.

13. И. ВИНИЦКИЙ, НЕЧТО О ПРИВИДЕНИЯХ: ИСТОРИИ О РУССКОЙ ЛИТЕРАТУРНОЙ МИФОЛОГИИ ХІХ ВЕКА, М. : МОСКОВСКИЙ КУЛЬТУРОЛОГИЧЕСКИЙ ЛИЦЕЙ, 1998, С. 69.

14. СМ.: R. Barthes, «L'effet de réel », Communications, $\mathrm{n}^{\circ} 11,1968$; И. БЕРЛИН, «ЁЖ И ЛИСА » (ПЕР. С АНГЛ. В. МИХАЙЛИНА), ИСТОРИЯ СВОБОДЫ: РОССИЯ, М.: НОВОЕ ЛИТЕРАТУРНОЕ ОБОЗРЕНИЕ, 2001, И ДР.

15. ОПИСАНИЕ ЭТИХ ФЕНОМЕНОВ СМ. ВО ВТОРОЙ И ТРЕТЬЕЙ ЧАСТЯХ КНИГИ: МИХАИЛ ЯМПОЛЬСКИЙ, ВОЗВРАЩЕНИЕ ЛЕВИАФАНА: ПОЛИТИЧЕСКАЯ ТЕОЛОГИЯ, РЕПРЕЗЕНТАЦИЯ ВЛАСТИ И КОНЕЦ СТАРОГО РЕЖИМА, М. : НОВОЕ ЛИТЕРАТУРНОЕ ОБОЗРЕНИЕ, 2004. 